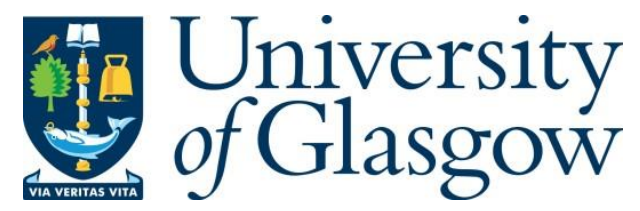

Teed, R. J. and Proctor, M. R. E. (2017) Quasi-cyclic behaviour in nonlinear simulations of the shear dynamo. Monthly Notices of the Royal Astronomical Society, 467(4), pp. 4858-4864. (doi:10.1093/mnras/stx421)

This is the author's final accepted version.

There may be differences between this version and the published version. You are advised to consult the publisher's version if you wish to cite from it.

http://eprints.gla.ac.uk/151406/

Deposited on: 09 November 2017

Enlighten - Research publications by members of the University of Glasgow http://eprints.gla.ac.uk 


\title{
Quasi-cyclic behaviour in non-linear simulations of the shear dynamo
}

\author{
Robert J. Teed ${ }^{1 \star}$ and Michael R. E. Proctor ${ }^{1} \dagger$ \\ ${ }^{1}$ Department of Applied Mathematics and Theoretical Physics, University of Cambridge, Cambridge, CB3 OWA, UK
}

Accepted XXX. Received YYY; in original form ZZZ

\begin{abstract}
The solar magnetic field displays features on a wide range of lengthscales including spatial and temporal coherence on scales considerably larger than the chaotic convection which generates the field. Explaining how the Sun generates and sustains such large-scale magnetic field has been a major challenge of dynamo theory for many decades. Traditionally the 'mean-field' approach, utilising the well-known $\alpha$-effect, has been used to explain the generation of large-scale field from small-scale turbulence. However, with the advent of increasingly high-resolution computer simulations there is doubt as to whether the mean-field method is applicable under solar conditions. Models such as the 'shear dynamo' provide an alternative mechanism for the generation of large-scale field. In recent work we showed that while coherent magnetic field was possible under kinematic conditions (where the kinetic energy is far greater than magnetic energy), the saturated state typically displayed a destruction of large-scale field and a transition to a small-scale state. In this paper we report that the quenching of large-scale field in this way is not the only regime possible in the saturated state of this model. Across a range of simulations we find quasi-cyclic behaviour where largescale field is preserved and oscillates between two preferred lengthscales. In this regime the kinetic and magnetic energies can be of a similar order of magnitude. These results demonstrate that there is mileage in the shear dynamo as a model for the solar dynamo.
\end{abstract}

Key words: Sun: dynamo - MHD - magnetic fields - methods: numerical

\section{INTRODUCTION}

The chaotic motions of plasma within the Sun's convection zone create its magnetic field via dynamo action (Moffatt 1978). Small-scale turbulence (on the order of megametres) is able to generate magnetic field on similar lengthscales by the local random twisting and stretching of existing field lines (Schekochihin et al. 2004, 2007). However, the Sun also displays coherent magnetic structures on far larger scales (up to the size of the star itself) in particular sunspots which exhibit changes on the 11-year solar cycle. Production of large-scale magnetic field from small-scale motions has traditionally been explained using 'mean-field theory'; a flow with a global net helicity twists and stretches field lines via the ' $\alpha$-effect' to generate large-scale magnetic field (Moffatt 1978; Rädler \& Rheinhardt 2007). However, it is questionable whether the usual calculation of the $\alpha$-effect is applicable under solar conditions where the magnetic Reynolds

* E-mail: R.J.Teed@damtp.cam.ac.uk

$\dagger$ E-mail: mrep@cam.ac.uk number, measuring the strength of turbulence, is very large (Cattaneo \& Hughes 2006; Hughes \& Cattaneo 2008).

The continued improvement in numerical simulations of solar dynamics has contributed to doubt over mean-field theory leading to the development of alternative plausible dynamo mechanisms that generate large-scale field without the reliance on helicity (Courvoisier et al. 2009; Proctor 2007, 2012; Sridhar \& Singh 2014; Rogachevskii \& Kleeorin 2003; Brandenburg et al. 2008; Yousef et al. 2008a). Many of these studies incorporate a large-scale velocity shear; the pioneering work of Yousef et al. (2008a) used forced, non-helical motion in domains with large aspect ratios. This set-up therefore excluded the $\alpha$-effect as a possible amplification mechanism and allowed for a more intensive exploration of parameter space than is possible using cubes. They found that large-scale magnetic field can be generated for sufficiently large shear with this 'shear dynamo' model. Several further studies confirmed and expanded upon the initial, kinematic results by: considering the effects of rotation Yousef et al. (2008b), deriving theoretical explanations (Heinemann et al. 
2011; McWilliams 2012), adding forced EMFs (Squire \& Bhattacharjee 2015a,b), and analysis of the saturated regime (Teed \& Proctor 2016).

The original study by Yousef et al. (2008a) considered only the kinematic regime of the problem, where the magnetic energy is much smaller than the kinetic energy and there is effectively no back reaction of the field on the flow. Previous studies with shear and zero helicity that have entered the nonlinear regime have shown that horizontally averaged mean field can be retained in after saturation (Brandenburg 2005; Brandenburg et al. 2008; Teed \& Proctor 2016). In particular, in a recent study (Teed \& Proctor 2016) we considered the saturated regime of the Yousef et al. (2008a) model (i.e. in boxes with large aspect ratios) for the first time allowing us to consider a wide parameter space. For the most part we found that the saturated regime is typified by a large increase in kinetic energy, the development of an additional large velocity shear, a quenching of the largescale field, and the appearance of small-scale field. However, a small subset of the simulations performed in that study demonstrated a secondary regime with different behaviour where large-scale field would persist, more akin to the coherent structures observed by Squire \& Bhattacharjee (2015b), where they forced EMFs directly.

Our new work discussed here expands upon the results of our previous study. With a more extensive study of parameter space we have established the robustness of the secondary regime found in Teed \& Proctor (2016) where the kinetic and magnetic energies equilibrate at values of similar order. In this regime the kinetic energy remains small enough to inhibit the manifestation of a fluctuation dynamo Schekochihin et al. (2004, 2007), which was postulated to be the cause of the quenched state previously observed. In the simulations discussed in this paper, coherent large-scale field persists deep into the saturated regime. The evolution of large-scale magnetic field can be quasi-periodic in nature as the solution oscillates between two different lengthscales. These results therefore help to restore faith in the (uniform) shear dynamo model as a tool for understanding the solar dynamo.

\section{METHODS}

The mathematical set-up is as described in Teed \& Proctor (2016) where we solve the incompressible magnetohydrodynamic (MHD) equations in the presence of a uniform shear flow, $\mathbf{U}=-S x \hat{\mathbf{y}}$, in a Cartesian shear-periodic box. The flow is forced with a white-noise non-helical homogeneous isotropic body force, $\mathbf{f}$, so the relevant equations are:

$$
\begin{aligned}
& \frac{d \mathbf{u}}{d t}=u_{x} S \hat{\mathbf{y}}-\frac{\nabla p}{\rho}+\frac{\mathbf{B} \cdot \nabla \mathbf{B}}{4 \pi \rho}+v \nabla^{2} \mathbf{u}+\mathbf{f}, \\
& \frac{d \mathbf{B}}{d t}=-B_{x} S \hat{\mathbf{y}}+\mathbf{B} \cdot \nabla \mathbf{u}+\eta \nabla^{2} \mathbf{B},
\end{aligned}
$$

where $\mathbf{u}$ and $\mathbf{B}$ are the velocity and magnetic fields respectively, and $d / d t=\partial_{t}-S x \partial_{y}+\mathbf{u} \cdot \nabla$. The effects of rotation and convective forcing are not considered in this model.

The parameter values used are $0.125 \leq S \leq 2,0.0075 \leq$ $v \leq 0.03$ and $0.005 \leq \eta \leq 0.013$ so that the magnetic Prandtl number, $P m=v / \eta$, takes values $0.75 \leq P m \leq 3$. We adopt units in which $L_{x}$ and $L_{y}$, the domain widths in the $x$ and $y$ directions, are set equal to unity. Forcing with constant mean amplitude is injected in a wavenumber shell centred at $k_{f} / 2 \pi=3$ so that the average forcing scale is $l_{f}=1 / 3$.

The equations are solved with shear-periodic boundary conditions (Umurhan \& Regev 2004; Lithwick 2007) using the code Snoopy (Lesur \& Longaretti 2005, 2007), which utilises a spectral method. The computational domain is elongated in the $z$-direction to ensure that the box is large enough to allow for a separation of scales. Therefore $L_{x}=1=L_{y}$ and $8 \leq L_{z} \leq 128$ so that $L_{z} \gg L_{x}, L_{y}$ with 32 points used for each unit of length. Modelling in boxes with large aspect ratios allows for a broad exploration of parameter space without being overly computational intensive. For instance, it is not currently computationally feasible to perform adequately resolved simulations at the Reynolds numbers needed in cubes of $128 \times 128 \times 128$.

Throughout we use the notation $\langle\cdot\rangle$ and $\cdot$ to indicate spatial and time averages respectively; subscripts on the angle brackets indicate an average over particular spatial coordinates.

\section{RESULTS}

Simulations are initialised with a weak seed field, $\langle B\rangle^{2} \sim$ $10^{-20}$, and allowed to grow (or, indeed, decay) through the kinematic (i.e. linear) regime. Confirmation of the results of the kinematic regime of the shear dynamo (i.e. the problem studied by Yousef et al. 2008a) using our code was presented in our previous work (Teed \& Proctor 2016), and was additionally independently verified by Squire \& Bhattacharjee (2015a). We therefore do not present results of the kinematic regime again here. The quantity $l_{B}$ defined by

$\frac{1}{l_{B}}=\overline{\left(\frac{\left\langle\left(\partial B_{y}^{<} / \partial z\right)^{2}\right\rangle_{z}}{\left\langle\left(B_{y}^{<}\right)^{2}\right\rangle_{z}}\right)^{1 / 2}}$,

gives an indication of the characteristic lengthscale of the mean field. An equivalent quantity, $l_{u}$, gives a definition for the characteristic lengthscale of the velocity field. Superscripts on these quantities indicate values that are calculated in the kinematic $(k)$ and saturated $(s)$ regimes.

\subsection{Quasi-cyclic behaviour}

The completion of the kinematic regime is achieved when the magnetic field saturates and the kinetic $\left(E_{K}\right)$ and magnetic $\left(E_{M}\right)$ energies equilibrate. This time is given by $t^{S}$ in Table 1 along with other input and output parameters for each simulation. Many simulations (runs A1-A10) exhibit what we deem to be 'quasi-cyclic' behaviour in the mean field, which will be explained below. The saturated values of the energies, and their ratio, indicate which regime (quenched or quasi-cyclic) is achieved by the final state of the solution. Fig. 1 shows the energies and their ratio for a typical simulation that displays quasi-cyclic behaviour in its saturated state. The magnetic energy grows during the kinematic phase, saturating (at time $t^{s} \sim 4 \times 10^{3}$ ) thereafter taking an average value of $E_{M} \sim 0.1$. Upon entering the saturated regime, the kinetic energy remains broadly unchanged from its kinematic value with an average value of $E_{K} \sim 0.8$. 


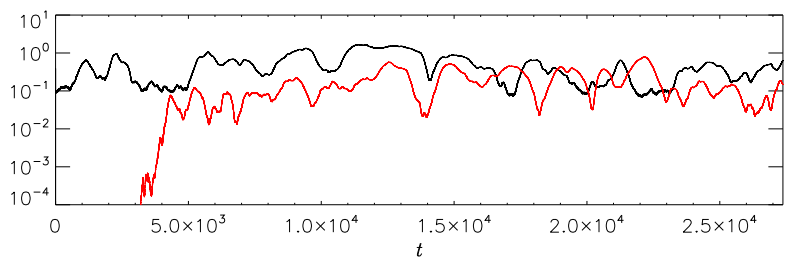

Figure 1. Kinetic energy, $E_{K}$ (black) and magnetic energy, $E_{M}$ (red) as a function of time for the run A3.

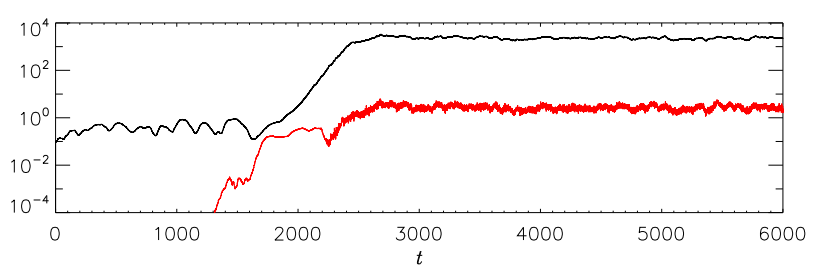

Figure 2. Kinetic energy, $E_{K}$ (black) and magnetic energy, $E_{M}$ (red) as a function of time for the run $\mathrm{C} 1$.

The energies therefore equilibrate with values of approximately the same order and this is more clearly seen in the ratio of the energies, $E_{K} / E_{M}$ which, upon saturation, obtains an $\mathcal{O}(1)$ value. The behaviour shown in Fig. 1 can be contrasted with that of Fig. 2, which shows the same quantities for a typical simulation where the mechanism that drives the large-scale magnetic field is quenched during saturation (run S2L16, see Teed \& Proctor 2016). Here the kinetic energy grows rapidly after the saturation of the magnetic field reaching a value that is $\sim 3$ orders of magnitude greater than the magnetic field. As discussed in Teed \& Proctor (2016) this coincides with the formation of a large-scale $z$-dependent shear flow in addition to the imposed $x$-dependent shear. This in turn leads to a quenching of large-scale field resulting in a saturated state dominated by small-scale magnetic structures.

It is clear from Figs. 1 and 2 that there exist (at least) two separate saturated states that the solution can obtain depending upon the choice of input parameters. In this paper we focus on the properties of the final state demonstrated by Fig. 1, which, as we shall see, retains large-scale field. For further details of the quenched state shown in Fig. 2, see Teed \& Proctor (2016).

Fig. 3 displays $\left\langle B_{y}\right\rangle_{x y}$, as a function of time and $z$ for run A8. Upon saturating $\left(\right.$ at $\left.t \sim 1.15 \times 10^{4}\right)$ the lengthscale of the magnetic field grows to fill the box $\left(L_{z}=16\right.$ in this case). This is also a feature of the quenched runs that was observed in our previous study. However, unlike the quenched runs the simulation shown in Fig. 3 show a persistence of large-scale magnetic field. In the saturated state the field fluctuates between periods of two different types of activity. One is typified by field on the scale of the box that wanders slowly in space (i.e. the field remains at near-constant $z$-values). This can be seen, for example, when $1.75 \times 10^{4} \leq t \leq 2.1 \times$ $10^{4}$. The second type of activity exhibits mean field on a scale smaller than the box that wanders in space, often very rapidly. One such period of activity can be seen, for example, during $1.5 \times 10^{4} \leq t \leq 1.7 \times 10^{4}$.

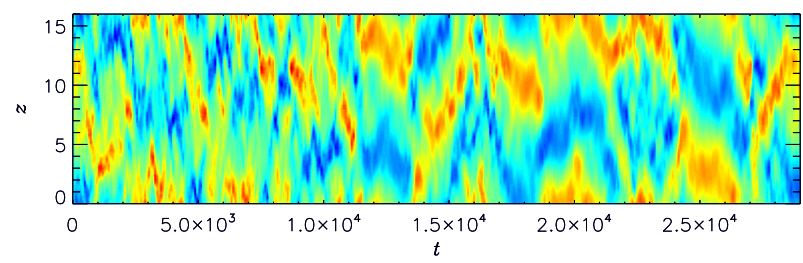

Figure 3. $B_{y}$, averaged over $x$ and $y$ and normalised using $B_{\mathrm{rms}}$, as a function of $z$ and $t$ for simulation A8.

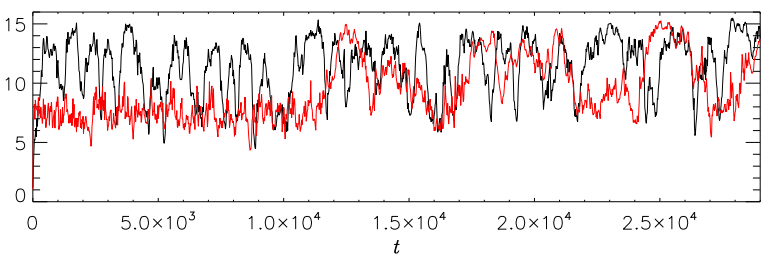

Figure 4. Velocity lengthscale, $l_{u}$ (black) and magnetic lengthscale, $l_{B}$ (red) for the run A8.

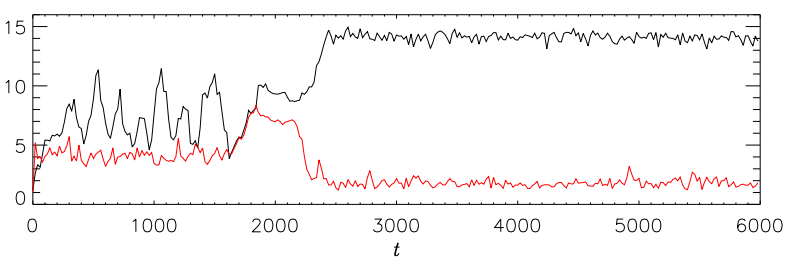

Figure 5. Velocity lengthscale, $l_{u}$ (black) and magnetic lengthscale, $l_{B}$ (red) for the run $\mathrm{C} 1$.

The two different lengthscales of the magnetic field can be observed in Fig. 4 . Periods where $l_{B} \sim 16$ (i.e. the scale of the box) are interrupted by typically briefer periods where $l_{B}$ is considerably smaller. In fact, during such times the lengthscale of the mean field returns to values seen during the kinematic regime. This indicates that these two scales in the saturated state are the box size and the intrinsic scale determined by the linear phase of the problem. In any simulation we define a quasi-cycle to be a period of time that contains a single instance of both $l_{B} \simeq L_{z}$ behaviour and $l_{B} \simeq l_{B}^{k}$ behaviour. For comparison, Fig. 5 shows the lengthscales in a quenched case (the same case as displayed in Fig. 2). Here the separation of scales in the saturated state is striking: the large velocity shear operates on the size of the box whereas the magnetic field is small-scale (considerably smaller than $\left.l_{B}^{k}\right)$.

The behaviour shown in Fig. 3, for run A3, has been observed across several simulations of varying shear rates and box sizes. For instance, Fig. 6, for run A7, shows the behaviour in another simulation with a larger box and different value of $\mathrm{Pm}$. The quasi-cycle is particularly evident in this run as evidenced by Fig. 7. Saturation occurs at $t \sim 9 \times 10^{3}$, after which the magnetic field gradually grows to the size of the box $\left(l_{B} \simeq 32\right)$ before equally slowly returning to a state where $l_{B} \simeq l_{B}^{k}$. The process then begins to repeat at $t \sim 1.8 \times 10^{4}$. This run also demonstrates two properties that 
Table 1. Table displaying the input and output parameters of the simulations performed in this study. Superscripts on $l_{B}$ indicate that the quantities were calculated for the kinematic regime $(\mathrm{k})$, the whole saturated regime $(\mathrm{s})$, the saturated regime when $l_{B}^{s} \simeq L_{z}(\mathrm{~L})$, and the saturated regime when $l_{B}^{s} \simeq l_{B}^{k}$, respectively. Runs are split into three sections in the table: i) the top section for runs displaying clear quasi-cyclic behaviour; ii) the middle section for runs displaying that are highly likely to be quasi-cyclic but have yet to complete a full quasi-cycle; iii) the bottom section for a run where large-scale magnetic field is very small (taken from Teed \& Proctor 2016).

\begin{tabular}{ccccccccccccccc}
\hline Run & $S$ & $L_{z}$ & $v$ & $\eta$ & $P m$ & $t^{s}$ & $l_{B}^{k}$ & $l_{u}^{k}$ & $\max \left(l_{B}^{s}\right)$ & $\min \left(l_{B}^{s}\right)$ & $\tau^{L}$ & $\tau^{l}$ & $l_{B}^{L}$ & $l_{B}^{l}$ \\
\hline A1 & 0.25 & 32 & 0.010 & 0.010 & 1.00 & 18000 & 11.01 & 18.06 & 30.55 & 13.83 & 4680 & 420 & 27.78 & 15.51 \\
A2 & 0.50 & 16 & 0.010 & 0.010 & 1.00 & 8500 & 7.64 & 9.03 & 15.76 & 5.36 & 2552 & 755 & 14.19 & 7.98 \\
A3 & 0.50 & 16 & 0.010 & 0.005 & 2.00 & 4300 & 5.03 & 8.86 & 14.96 & 5.18 & 560 & 127 & 13.41 & 6.85 \\
A4 & 0.50 & 16 & 0.020 & 0.010 & 2.00 & 28000 & 10.07 & 11.14 & 15.10 & 6.86 & 2660 & 1513 & 13.72 & 10.07 \\
A5 & 0.50 & 16 & 0.010 & 0.013 & 0.75 & 30000 & 8.96 & 9.26 & 14.68 & 6.54 & 700 & 2050 & 14.20 & 9.91 \\
A6 & 0.50 & 16 & 0.0075 & 0.010 & 0.75 & 5250 & 7.14 & 9.00 & 15.52 & 5.78 & 1603 & 333 & 12.96 & 8.38 \\
A7 & 0.50 & 32 & 0.010 & 0.013 & 0.75 & 9000 & 10.23 & 15.40 & 29.64 & 7.94 & 2840 & 400 & 28.21 & 10.00 \\
A8 & 1.00 & 16 & 0.020 & 0.010 & 2.00 & 11500 & 7.46 & 10.81 & 15.24 & 5.44 & 1607 & 900 & 12.81 & 8.66 \\
A9 & 2.00 & 16 & 0.020 & 0.010 & 2.00 & 7500 & 5.46 & 9.05 & 15.18 & 5.54 & 720 & 295 & 12.14 & 8.09 \\
A10 & 2.00 & 16 & 0.030 & 0.010 & 3.00 & 9000 & 7.53 & 11.01 & 15.33 & 4.79 & 1665 & 352 & 13.75 & 8.14 \\
\hline B1 & 0.125 & 64 & 0.010 & 0.01 & 1.00 & 35000 & 12.84 & 31.16 & - & - & - & - & - & - \\
B2 & 0.25 & 64 & 0.010 & 0.01 & 1.00 & 14000 & 10.24 & 29.69 & - & - & - & - & - & - \\
B3 & 0.25 & 128 & 0.010 & 0.01 & 1.00 & 12000 & 10.57 & 36.83 & - & - & - & - & - & - \\
B4 & 0.5 & 64 & 0.010 & 0.01 & 1.00 & 5000 & 7.99 & 17.04 & - & - & - & - & - & - \\
B5 & 0.5 & 128 & 0.010 & 0.01 & 1.00 & 5000 & 7.86 & 21.11 & - & - & - & - & - \\
\hline C1 & 2.00 & 16 & 0.010 & 0.010 & 1.00 & 1750 & 4.11 & 6.94 & 8.35 & 1.21 & - & - & - \\
\hline
\end{tabular}

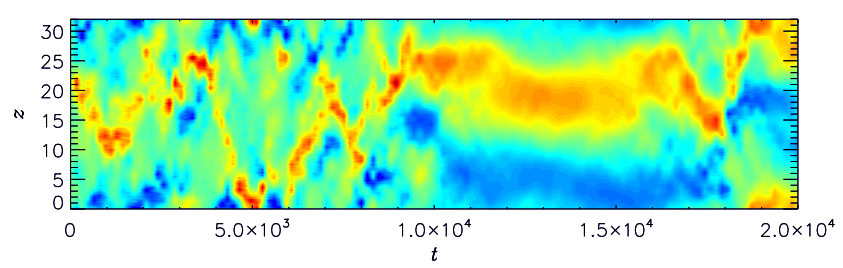

Figure 6. $B_{y}$, averaged over $x$ and $y$ and normalised using $B_{\mathrm{rms}}$, as a function of $z$ and $t$ for simulation A7.

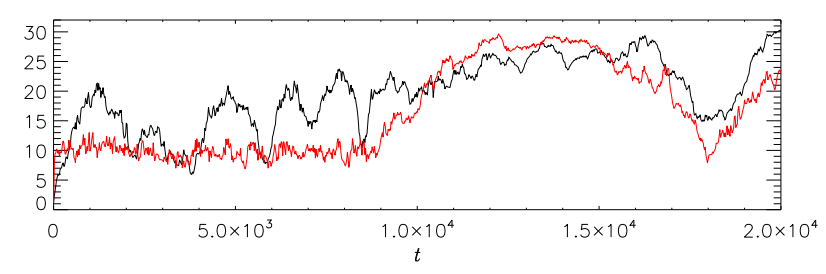

Figure 7. Velocity lengthscale, $l_{u}$ (black) and magnetic lengthscale, $l_{B}$ (red) for the run A7.

are typical across the suite of runs. First, the magnetic field wanders very slowly when $l_{B} \simeq L_{z}$ demonstrated by the near constant $z$-locations of the field in Fig. 6. Second, periods where $l_{B} \simeq l_{B}^{k}$ can be extremely brief compared to periods with $l_{B} \simeq L_{z}$, evidenced in Fig. 7 .

In addition to runs that display clear and persistent quasi-cyclic behaviour, several further simulations (runs B1B5) are highly likely to be quasi-cyclic in nature; these are shown in the middle section of Table 1. Upon saturation all of these runs exhibit large-scale field on a scale larger than that of the kinematic regime for the remainder of the simulation. In all cases we have integrated into the saturated regime for at least as long as the kinematic phase. Since these runs are amongst the most computationally intensive runs we have performed, it has not yet been possible to reach the end of a quasi-cycle (assuming such phenomena exist in these runs!). However, although we cannot categorically state that quasi-cycles will appear, we can say with near certainty that the mechanism generating large-scale field will not quench in these runs. This is because, unlike runs from Teed \& Proctor (2016) that exhibit quenching, the kinetic energy here remains of the same order as the kinematic regime.

\subsection{Quantifying length- and time-scales}

Given that two lengthscales clearly exist in the saturated states observed in Figs. 3-7, it is desirable to quantify both the lengthscales and the time spent in each configuration. In order to do this we define several quantities, the values of which are displayed in Table 1 . The values $\max \left(l_{B}^{s}\right)$ and $\min \left(l_{B}^{s}\right)$ are simply the maximum and minimum values that $l_{B}$ takes during the saturated regime (i.e. for $t>t^{S}$ ).

Using the time series data of $l_{B}$ we also calculate times for which $d l_{B} / d t \sim 0$ since this indicates periods when the lengthscale of the magnetic field is approximately constant. During such periods we then determine whether $l_{B}$ falls within a certain range of values. These intervals are based on fractions of $L_{z}$ and $l_{B}^{k}$ and stipulate that either $-l_{B}^{k} / 4 \leq$ $l_{B}-l_{B}^{k} \leq l_{B}^{k} / 4$ or $-L_{z} / 4 \leq l_{B}-L_{z}$. This method provides a measure of periods of the simulation when the magnetic field is contained within one of its two chosen regimes (i.e. extended periods when $l_{B}^{s} \sim l_{B}^{k}$ or when $l_{B}^{s} \sim L_{z}$ ). The values $\tau^{L}$ and $\tau^{l}$ are the average periods of time spent in the $l_{B}^{S} \sim L_{z}$ and the $l_{B}^{s} \sim l_{B}^{k}$ regime, respectively. Likewise the values $l_{B}^{L}$ and $l_{B}^{l}$ are the averages of $l_{B}$ calculated during the respective time periods.

The measures of the lengthscale of the magnetic field shown in both Table 1 and Fig. 8 demonstrate the two lengthscales across the suite of simulations. Fig. 8a shows 
that the maximum value of $l_{B}^{s}$ achieved is always on the scale of the box ( $\simeq 16$ for most runs) whilst the minimum value is linearly dependent on $l_{B}^{k}$, the lengthscale in the kinematic regime. The latter indicates that the smaller of the two scales observed in the saturated state is indeed the intrinsic scale of the kinematic problem. This is borne out in Fig. 8b where $l_{B}^{l} \simeq l_{B}^{k}$ across a range of simulations. There also appears to be a slight linear dependence of $l_{B}^{L}$ (the large lengthscale) on the kinematic lengthscale, at least for runs with $L_{z}=16$ (there are too few points to determine the behaviour when $\left.L_{z}=32\right)$.

The average period of time spent during an episode of each magnetic scale is also linearly dependent on $l_{B}^{k}$ as shown in Fig. 9. Other than a few outlying points both $\tau^{l}$ and $\tau^{L}$ scale linearly with the intrinsic magnetic scale of the kinematic regime. In general the periods spent when $l_{B}^{S} \simeq L_{z}$ are longer than those when $l_{B}^{s} \simeq l_{B}^{k}$. Assuming that a perturbation of some sort is required to move between the two regimes, this indicates that larger magnetic scales are more stable to such transitions. Since both time periods scale linearly with the intrinsic magnetic scale, then, consequently, the average period of a 'quasi-cycle' does so also. This quantity is measured by $\tau^{L}+\tau^{l}$ and is also shown in Fig. 9. The periods of the quasi-cycles range from $\sim 1000$ to $\sim 5000$ in this suite of runs.

Data in Figs. 8 and 9 are plotted against $l_{B}^{k}$, rather than the input parameters of the system (i.e. $S, v, \eta$ or $\mathrm{Pm}$ ), because of the clear dependence it demonstrates. Plots of parameters measuring the saturated regime against input parameters do not show such clear correlation. This is because, although the quasi-cyclic state has been observed across a range of $S, v$ and $\eta$, the simulations are performed using a small number of distinct values of these parameters. Broadly speaking the linear dependence on $l_{B}^{k}$ of both the magnetic lengthscales and periods of quasi-cycles translates to a $S^{-1 / 2}$ dependence on shear, as expected from the known kinematic results where $l_{B}^{k} \sim S^{-1 / 2}$ (Yousef et al. 2008a). We are currently unable to identify a clear dependence on the diffusion parameters, or even on $P m=v / \eta$, which is further complicated because any distinct value of $P m$ can be formed by infinitely-many different combinations of $v$ and $\eta$. The identification of further runs with quasi-cyclic behaviour is required to fully understand the dependence of the state on the shear and the diffusion parameters. This should be conducted as part of a broader study that also determines the dependence of the manifestation of the quasi-cyclic versus quenched state on $S, v$ and $\eta$. This is work in progress.

\subsection{Kinetic and magnetic correlation}

Some simulations show strong correlation between both the energies and lengthscales of the velocity and magnetic fields which allows the quasi-cycle to be examined in further detail. Fig. 10 displays the energies for such a run where growth in kinetic energy is frequently curtailed shortly after a drop in the magnetic energy. For example, at $t \sim 1.48 \times 10^{4}$, the magnetic energy suffers a sharp reduction shortly followed by a similar fall in kinetic energy. This modification of the velocity by the magnetic field appears to stop run-away growth of the kinetic energy that would otherwise lead to a quenched state such as that seen in Fig. 2. The behaviour is also observed in the lengthscales of the two fields evidenced by

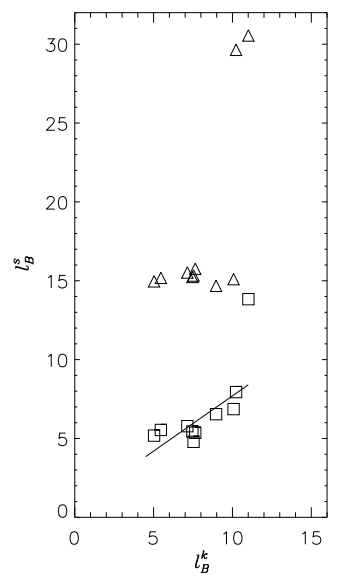

(a)

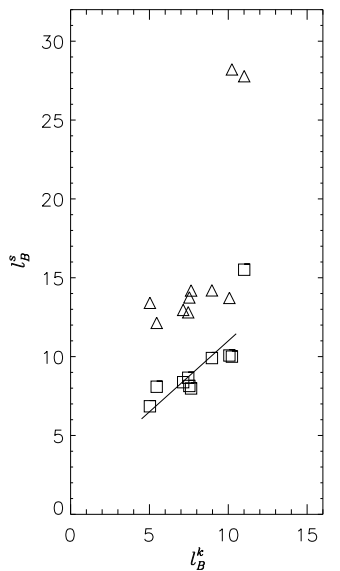

(b)
Figure 8. Plots showing (a) the extrema values and (b) the averaged values of $l_{B}^{s}$ (the lengthscale of the magnetic field in the saturated regime) against the intrinsic magnetic scale from the kinematic regime, $l_{B}^{k}$, for our suite of runs. In (a) triangles and squares represent the maximum and minimum values obtained by $l_{B}^{s}$, respectively. In (b) triangles and squares represent the values of $l_{B}^{L}$ and $l_{B}^{l}$, respectively.

Figs. 11 and 12. A dramatic drop in the scale of magnetic field (at $t \sim 1.48 \times 10^{4}$ ) is immediately followed by an equivalent drop in the scale of the velocity. This demonstrates the magnetic field's ability to influence the scale of the flow via the Lorentz forces in this quasi-cyclic saturated regime. Conversely, in the quenched state the magnetic field is effectively a slave to the velocity because of the large disparity in the magnitudes of the energies and hence the dominance of inertia over Lorentz forces.

The cause of the initial drop in magnetic lengthscale while the velocity lengthscale remains large is unclear. However, the subsequent drop in velocity lengthscale allows the magnetic scale to grow to fill the box, in a repeat of the process seen at the very start of the saturated regime. This, in turn, allows new growth of flows on the size of the box and a steady increase in kinetic energy. At $t \sim 1.75 \times 10^{4}$ the process begins to repeat with a new sharp reduction in magnetic energy and lengthscale. Another clear feature seen in Fig. 11 is the reversal of magnetic field with each quasi-cycle. Locations of positive field when $l_{B}^{S} \simeq L_{z}$ are replaced with negative field in a new quasi-cycle and vice-versa. This occurs because of the fast migration of field during each short-term $l_{B}^{s} \simeq l_{B}^{k}$ event.

The sort of quasi-periodic behaviour demonstrated in run A6 is reminiscent of features seen in other contexts of (magneto)hydrodynamics. One such example, in rotating (non-magnetic) convection, is a competition between shear flows and convection known as 'convective bursts' (Morin \& Dormy 2004; Teed et al. 2012). In this case convection generates strong zonal flows through the Reynolds stresses. These shearing flows then inhibit the convection and hence reduce the source of energy for themselves, returning the system to a convective state whereby the cycle repeats. We postulate that a similar mechanism may exist in this shear dynamo model as the magnetic field oscillates between its two in- 


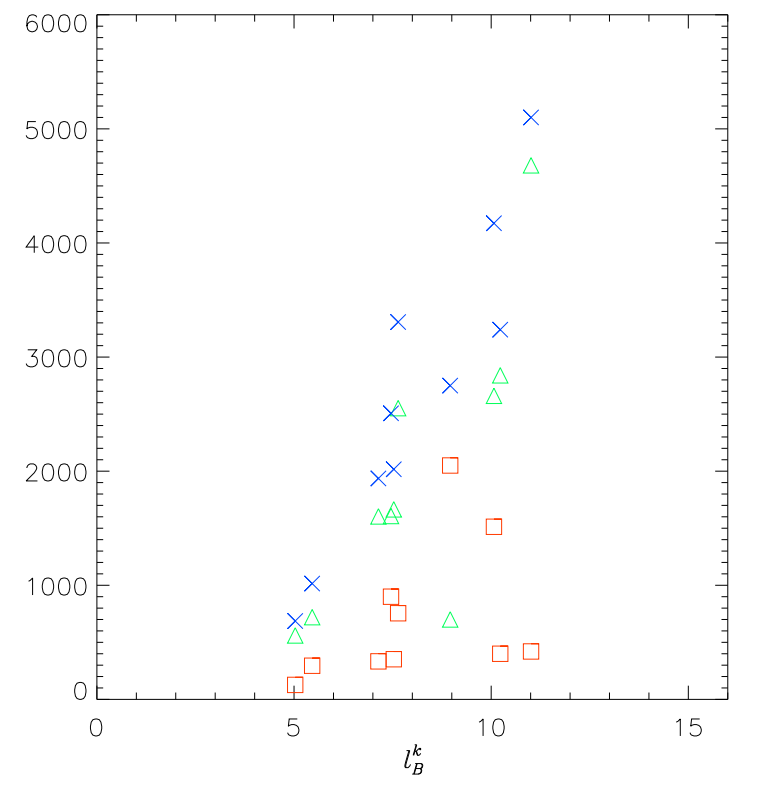

Figure 9. Plot showing the average length of time spent in each part of the quasi-cycle for our suite of runs. Green triangles represent $\tau^{L}$ (the period of $L_{B}^{s} \simeq L_{z}$ behaviour) and red squares $\tau^{l}$ (the period of $l_{B}^{s} \simeq l_{B}^{k}$ behaviour). Also plotted is $\tau^{L}+\tau^{l}$, the average length of a quasi-cycle, represented by blue crosses.

trinsic lengthscales of kinematic and saturated states. Upon saturation it is clear from all runs that we have performed that the magnetic field first attempts to reach a new preferred lengthscale (different to that of the kinematic phase) and this in turn produces a growing large shear flow on the scale of the box. It is feasible that this large-scale shearing flow is of a similar nature to that described by Käpylä et al. (2009). This flow can appear spontaneously in nonmagnetic calculations with a large imposed shear by a process referred to as the 'vorticity dynamo'. However, amongst our nonmagnetic simulations (not shown here) we observe some instances when this flow emerges and others where it does not. It is also of note that in our MHD runs the large-scale flow never materialises during the kinematic phase of the problem; i.e. when the field is weak. This leads us to believe that the manifestation of the flow is, at least in some parameter regimes, dependent on the existence of magnetic field in the problem.

Without modification the shearing flow grows in magnitude and can ultimately destroy large-scale field, as we observed in Teed \& Proctor (2016). However, a drop in magnetic scale back to the kinematic lengthscale, $l_{B}^{k}$, leads to the generation of smaller flows via the Lorentz forces inhibiting the shearing flow. Indeed, magnetic fields tend to suppress the vorticity dynamo (Käpylä \& Brandenburg 2009). Therefore a strong enough magnetic field in the saturated state could curtail the growth of the shearing flow if it is formed by such a mechanism. Once the flow scale is adequately reduced, the magnetic field can then grow to a preferred saturated state lengthscale, which is at least the size of the box. The process then repeats, thus frequently regulating kinetic energy growth so that $E_{K} / E_{M} \sim \mathcal{O}(1)$. It is unclear

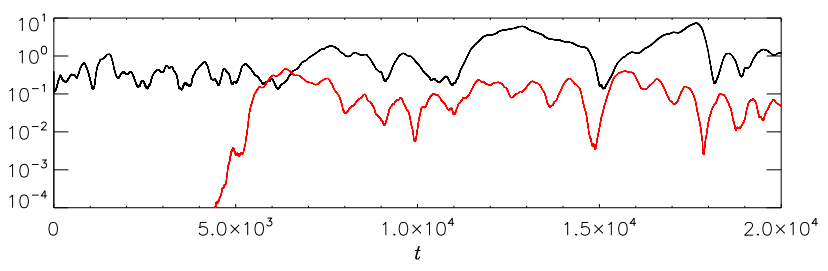

Figure 10. Kinetic energy, $E_{K}$ (black) and magnetic energy, $E_{M}$ (red) as a function of time for the run A6.

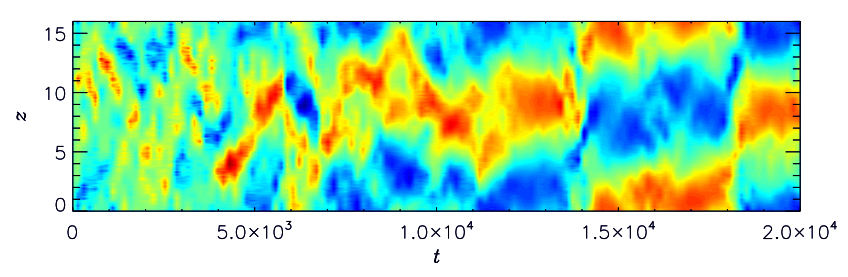

Figure 11. $B_{y}$, averaged over $x$ and $y$ and normalised using $B_{\mathrm{rms}}$, as a function of $z$ and $t$ for simulation A6.

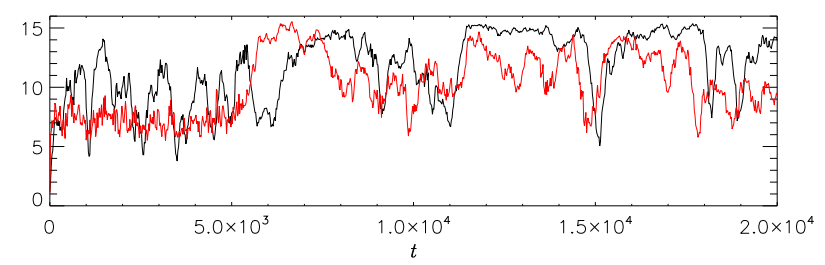

Figure 12. Velocity lengthscale, $l_{u}$ (black) and magnetic lengthscale, $l_{B}$ (red) for the run A6.

in this process what exactly instigates the initial reduction of the magnetic lengthscale and why this occurs under some parameter regimes but not those of the previous quenched runs.

\section{CONCLUSIONS}

The retention of large-scale magnetic field in the saturated state discussed in this work indicates that the shear dynamo model in its basic form (i.e. using a uniform shear profile with non-helical forcing) can potentially form the basis of a model for the solar dynamo. This is an improvement on our previous work (Teed \& Proctor 2016) where the outlook was less promising because the magnetic field was found to saturate as a small-scale dynamo in almost all runs performed. The extension of parameter space into models with $P m \neq 1$ has allowed us to observe the second regime across a range of simulations.

The magnetic field of the quasi-cyclic regime exhibits two lengthscales: one on the size of the box and another on the intrinsic scale of the kinematic regime. It is the transition between these scales that creates a quasi-periodic behaviour as the system moves between periods with $l_{B}^{S} \simeq L_{z}$ and $l_{B}^{S} \simeq l_{B}^{k}$. Periods when the mean field is on the scale of the box tend to be longer than the interruptions by smaller-scale mean field. 
An outstanding question is whether the larger of the two magnetic scales observed has an intrinsic size that is restricted by the scale of the boxes used. In the runs exhibiting unquestionably quasi-cyclic behaviour the field always fills the box. However, amongst runs where quasi-cycles are yet to be completed, there is tentative evidence that the field may be saturating at a scale smaller than the box. If this is the case it indicates that the magnetic field has a parameterdependent preferred lengthscale in both the kinematic and saturated regimes, although the values differ greatly. Conversely, it may simply be the case that the simulations (in these computationally expensive large boxes) have yet to be run long enough for the magnetic scale to reach the box size. Continuation of these runs, as well as initialisation of new runs, is required to resolve this issue.

The conditions that determine which final state (quasicyclic or quenched) is selected are not well understood. However, our results suggest that the kinetic and magnetic energies must equilibrate within approximately an order of magnitude of one another to avoid development of a state where the magnetic field is small-scale. Previous results, including one run retained here from Teed \& Proctor (2016), show that if the kinetic energy is able to grow rapidly without hindrance at saturation then a small-scale dynamo develops. Conversely, in the quasi-cyclic regime the kinetic energy is restricted by the magnetic field which can regularly halt its growth as the solution also adjusts its lengthscale. One possible explanation is that the observed quasi-cyclic behaviour operates as relaxation oscillations between a vorticity dynamo (for the velocity) and a shear dynamo (for the magnetic field). In this scenario the large $z$-dependent shearing flow would be generated by a vorticity dynamo when the field is weak (Käpylä et al. 2009). However, stronger magnetic field would suppress this mechanism (Käpylä \& Brandenburg 2009), resulting in a weak vertical shear and a shear dynamo could operate efficiently. This can only occur if the kinetic and magnetic energies are of a similar order in the saturated state. Simulations where this is not the case and small-scale field arises (Teed \& Proctor 2016) would then be situations where the vorticity dynamo greatly dominates and no large-scale field can be generated by a shear dynamo mechanism. In this case the (weak) magnetic field is generated by a fluctuation dynamo mechanism and hence its lengthscale is reduced to that of the imposed forcing. A systematic survey - including with different box sizes - is required to establish the parameter space that admits each type of saturated state. Such a survey is hindered by the expensive nature of the simulations but nevertheless is work in progress.

The role of shear in dynamo models aiming to explain the manifestation of large-scale field in the Sun and other astrophysical bodies can be abstruse. Even in the kinematic regime other models demonstrate conflicting roles for the shear depending on the exact set-up. Tobias \& Cattaneo (2013) show that a sinusoidal shear bolsters large-scale field through the suppression of the small-scale dynamo, yet Sood et al. (2016) find (in a spherical model) that the addition of shear suppresses dynamo action. We have seen that the (relatively) simple shear used in our model can either promote or inhibit the production of large-scale field (in the saturated state) depending on the input parameters. The quasi-cyclic behaviour observed is hard to identify with the solar cycle directly. However, the fact that spatially wandering largescale field with quasi-cyclic behaviour (including field reversals) can be generated in the fully evolved state represents a major advance. This knowledge encourages continued investigation of shear dynamo models. Moreover, given the ubiquitous nature of shears throughout astrophysical bodies exhibiting magnetic fields, it is very desirable to determine the conditions under which shear either encourages or suppresses large-scale dynamo action.

Further tweaking of our model's set-up to promote more solar-like behaviour such as periodic cycles could involve several ideas. These include, but are not limited to, the consideration of: i) different shear profiles, ii) inhomogeneous shear profiles that vary in the long direction, iii) forcing with a small degree of helicity. In various ways these tweaks could promote spatially and temporally wandering large-scale field that is periodic in nature. Indeed, one such study using a 2.5D model has shown periodic behaviour in the form of dynamo waves Tobias \& Cattaneo (2013). These ideas will be the focus of future investigations into using shear driven dynamos to model the solar dynamo and solar cycle.

\section{ACKNOWLEDGEMENTS}

The authors wish to thank the reviewer, Dr. Axel Brandenburg, for constructive comments which improved the quality of our manuscript. This work was supported by the Science and Technology Facilities Council, grant ST/L000636/1.

\section{REFERENCES}

Brandenburg A., 2005, The Astrophysical Journal, 625, 539

Brandenburg A., K.-H. Rädler K.-H., Rheinhardt M., Käpylä P., 2008, The Astrophysical Journal, 676, 740

Cattaneo F., Hughes D. W., 2006, Journal of Fluid Mechanics, 553,401

Courvoisier A., Hughes D. W., Tobias S. M., 2009, Journal of Fluid Mechanics, 627, 403

Heinemann T., McWilliams J., Schekochihin A., 2011, Physical review letters, 107, 255004

Hughes D. W., Cattaneo F., 2008, Journal of Fluid Mechanics, 594,445

Käpylä P. J., Brandenburg A., 2009, The Astrophysical Journal, 699, 1059

Käpylä P. J., Mitra D., Brandenburg A., 2009, Phys. Rev. E, 79, 016302

Lesur G., Longaretti P.-Y., 2005, Astronomy \& Astrophysics, 444, 25

Lesur G., Longaretti P.-Y., 2007, Monthly Notices of the Royal Astronomical Society, 378, 1471

Lithwick Y., 2007, The Astrophysical Journal, 670, 789

McWilliams J. C., 2012, Journal of Fluid Mechanics, 699, 414

Moffatt H. K., 1978, Field Generation in Electrically Conducting Fluids. Cambridge University Press, Cambridge, London, New York, Melbourne

Morin V., Dormy E., 2004, Phys. Fluids, 16, 1603

Proctor M. R. E., 2007, Monthly Notices of the Royal Astronomical Society: Letters, 382, L39

Proctor M. R. E., 2012, Journal of Fluid Mechanics, 697, 504

Rädler K.-H., Rheinhardt M., 2007, Geophysical \& Astro Fluid Dynamics, 101, 117

Rogachevskii I., Kleeorin N., 2003, Physical Review E, 68, 036301 


\section{$8 \quad$ R. J. Teed and M. R. E. Proctor}

Schekochihin A. A., Cowley S. C., Taylor S. F., Maron J. L., McWilliams J. C., 2004, The Astrophysical Journal, 612, 276 Schekochihin A., Iskakov A., Cowley S., McWilliams J., Proctor M. R. E., Yousef T., 2007, New Journal of Physics, 9, 300 Sood A., Hollerbach R., Kim E.-J., 2016, Journal of Physics A: Mathematical and Theoretical, 49, 425501

Squire J., Bhattacharjee A., 2015a, Physical review letters, 115, 175003

Squire J., Bhattacharjee A., 2015b, The Astrophysical Journal, 813,52

Sridhar S., Singh N. K., 2014, Monthly Notices of the Royal Astronomical Society, 445,3770

Teed R. J., Proctor M. R. E., 2016, Monthly Notices of the Royal Astronomical Society, 458, 2885

Teed R. J., Jones C. A., Hollerbach R., 2012, Phys. Fluids, 24, 066604

Tobias S., Cattaneo F., 2013, Nature, 497, 463

Umurhan O. M., Regev O., 2004, Astronomy \& Astrophysics, 427, 855

Yousef T. A., Heinemann T., Schekochihin A. A., Kleeorin N., Rogachevskii I., Iskakov A. B., Cowley S. C., McWilliams J. C., 2008a, Physical review letters, 100, 184501

Yousef T. A., Heinemann T., Rincon F., Schekochihin A., Kleeorin N., Rogachevskii I., Cowley S. C., McWilliams J. C., 2008b, Astronomische Nachrichten, 329, 737

This paper has been typeset from a $\mathrm{T}_{\mathrm{E}} \mathrm{X} / \mathrm{LA} \mathrm{T}_{\mathrm{E}} \mathrm{X}$ file prepared by the author. 